\title{
La inmigración estudiantil y la adaptación escolar.
}

\section{Student immigration and school adaptation.}

Pimbo Tibán Ana Gabriel ${ }^{1}$., Canchignia Bonifaz Ana Lucía ${ }^{2}$., Paredes Hernández Nelly Elizabeth ${ }^{3}$. \& Yungán Yungán Raúl ${ }^{4}$.

\begin{abstract}
DOI: https://doi.org/10.33262/cienciadigital.v9i2.385

This article includes the study on student immigration and school adaptation; the processes of access and integration to the educational institutions of Ecuador of immigrant children and adolescents; Glimpsing a big problem that lies in the presence of a decontextualized curriculum and the absence of curricular adaptations by teachers; before the ethnic and cultural diversity of immigration. The theoretical justification is the bibliographic compilation of multiple authors who have made different investigations in multiple contexts that speak about student immigration and school adaptation. Therefore, the objective of this research is to show how immigrant students were integrated, how they adapted to the cultural diversity of Ecuador and whether various curricular adaptations were made so that they could be included in the educational system. The application of the survey was investigated as a technique and the use of a questionnaire as an instrument aimed at immigrant students and teachers of the Educational Unit "Teresa Flor" in the city of Ambato, Tungurahua province; in the school year 2018 - 2019.school year 2018 - 2019.
\end{abstract}

Keywords: Immigration, curriculum, curriculum adaptation and cultural diversity.

\footnotetext{
${ }^{1}$ Universidad Técnica de Amato, Facultad de Ciencias de la Educación, Carrera de Educación Básica. Ambato, Ecuador.apimbo0329@uta.edu.ec

${ }^{2}$ Universidad Técnica de Amato, Facultad de Ciencias de la Educación, Carrera de Educación Básica. Ambato, Ecuador. acanchignia5048@uta.edu.ec

${ }^{3}$ Universidad Técnica de Amato, Facultad de Ciencias de la Educación, Carrera de Educación Básica. Ambato, Ecuador. nparedes4648@uta.edu.ec

${ }^{4}$ Universidad Técnica de Amato, Facultad de Ciencias de la Educación, Carrera de Educación Básica. Ambato, Ecuador. ryungan@uta.edu.ec
} 


\section{Resumen}

El presente artículo comprende el estudio sobre la inmigración estudiantil y la adaptación escolar; los procesos de acceso e integración a las instituciones educativas del Ecuador de los niños, niñas y adolescentes inmigrantes; Vislumbrando un gran problema que radica en la presencia de un currículo descontextualizado y la ausencia de adaptaciones curriculares por parte de los docentes; ante la diversidad étnica y cultural de la inmigración. La justificación teórica es la recopilación bibliográfica de múltiples autores que han realizado diferentes investigaciones en múltiples contextos que hablan acerca de la inmigración estudiantil y la adaptación escolar. Por lo tanto, el objetivo de esta investigación es dar a conocer como los estudiantes inmigrantes se integraron, la manera en la que se adaptaron ante la diversidad cultural del Ecuador y si se realizaron diversas adaptaciones curriculares para que lograsen ser incluidos en el sistema educativo. Se indagó mediante la aplicación de la encuesta como técnica y la utilización de un cuestionario como instrumento dirigido a los estudiantes inmigrantes y a los docentes de la Unidad Educativa "Teresa Flor" en la ciudad de Ambato, provincia de Tungurahua; en el periodo lectivo 2018 - 2019.

Palabras clave: Inmigración, currículo, adaptación curricular y diversidad cultural.

\section{Introducción.}

La conceptualización de la inmigración estudiantil y la adaptación escolar ha sido una problemática que obliga al estado de cada país y a la sociedad en general a restructurar el sistema educativo que permita el acceso de todos los niños, niñas y adolescentes ya sean autóctonos o inmigrantes. La inmigración es un proceso complejo especialmente para los niños pues tienen que abandonar muchas cosas en su país de origen; a sus amigos, juguetes, abuelos, entre otras cosas; llevándolos a enfrentarse a un nuevo mundo en donde no conocen nada; ni a nadie.

En el ámbito educativo los estudiante inmigrantes se enfrentan a un proceso de ansiedad pues les invade el temor a ser "los nuevos del salón", al idioma, a aprender asignaturas desconocidas y a hacer nuevos amigos; es aquí en donde se da la importancia a alcanzar la adaptación escolar que permita que los estudiantes que provienen de otros países puedan acceder a la educación en el marco de la igualdad de derechos antes los estudiantes autóctonos; una educación que les provea de seguridad, paz, empatía y que respete su diversidad cultural y ética.

Pero se debe analizar si el sistema educativo de cada país está preparado para ofrecer aquella educación. La presencia de migrantes internacionales en las escuelas, muestra que el sistema educativo está diseñado para estudiantes que no migran puesto que sus políticas educativas, contenidos y programas son tan centralizados. Por lo tanto, obliga al Estado y al sistema educativo a repensar y cambiar aspectos en cuanto a la formación inicial de 
los docentes, actualización de los maestros, selección de contenidos y pautas de evaluación y acceso escolar. (Zúñiga, 2013)

Se reconoce que los estudiantes binacionales y transnacionales no existen en los programas de formación inicial docentes, en los diferentes programas de actualización del magisterio, en los diseños curriculares, ni en los planes y políticas educativas. De manera que se ignora la realidad y proveniencia de los estudiantes. (Zúñiga, 2013)

Por ello la presencia de niños, niñas y adolescentes inmigrantes en el sistema educativo genera grandes retos y desfíos para el Estado. En la investigación realizada por Cerón, Pérez Alvarado, \& Poblete, (2017) afirman que el sistema educativo y en especial cada una de las instituciones educativas reciben a estudiantes extranjeros en busca del logro de la inclusión y sustenta que el docente juega un rol importante en el logro de dicha inclusión puesto que, la percepción que estos tengan de los estudiantes inmigrantes fortalecerá el camino hacia la construcción de una cultura inclusiva (Cerón, Pérez Alvarado, \& Poblete, 2017).

El protagonismo docente radica en la relevancia que tienen los docentes en el proceso de inclusión al centro educativo de los estudiantes inmigrantes; cuán importante es considerar las visiones que estos tengan de sus estudiantes ya que estas visiones llegarían a ser potencialmente inclusivas o exclusivas. Los docentes tienen una visón restringida en cuanto al tema de la inclusión puesto que consideran a los migrantes como personas que "presentan una serie de déficits académicos y de comportamiento que dificultan su proceso de inclusión y su plena participación en las escuelas" (Cerón, Pérez Alvarado, \& Poblete, 2017). Por lo tanto, Cerón, Pérez Alvarado, \& Poblete (2017) hacen un llamado a que se reflexione sobre los procesos de formación inicial docente; repensar y mejorar dichos procesos de manera que permitan fortalecer sus competencias y visualicen a los migrantes no desde una cultura folclórica sino desde un instrumento para el aprendizaje entre culturas

Otro aspecto importante es considerar, si el currículo es abierto y flexible ante la diversidad que se pueda presentar dentro del quehacer educativo, dentro de las aulas; en las escuelas con presencia de niños y niñas migrantes. La complejidad de desarrollo del currículo educativo en contextos de estudiantes migrantes, radica en que los principales países de destinos migratorios deben ampliar la diversidad en el ámbito educativo. Pero a partir de esta premisa no se logra eliminar conductas discriminatorias y racistas por parte de los actores de la comunidad educativa, y el currículo educativo aún sigue siendo descontextualizado. Por lo tanto, para lograr la adaptación escolar el estado debe abordar políticas educativas que fomenten propuestas curriculares interculturales permitirá la aceptación de la diversidad ética - cultural de la migración (Hernández Yulcerán, 2016)

Los docentes deben conocer las cualidades que poseen los distintos grupos culturales y utilizarlos como medios para el enriquecimiento de la clase; de manera que promuevan la participación de todos los estudiantes independientemente de su nacionalidad. Este medio propicia la igualdad educativa; otorgando las mismas oportunidades de aprendizaje de 
todos y cada uno de los niños. (Hernández Yulcerán, 2016)

Poblete, (2018) afirma que existe una discusión en torno al currículo escolar y la procedencia de los estudiantes, las adaptaciones que se puede realizar con el fin de garantizar la adquisición de habilidades y destrezas que los educandos logren adquirir; obteniendo capacidades que les permita desenvolverse en el mundo global. Por lo tanto, sustenta que el estado actual de la sociedad en el ámbito cultural y multicultural demanda repensar sobre; qué se enseña y cómo se enseña proponiendo así la renovación pedagógica.

Los establecimientos que cuentan con estudiantes extranjeros, deben garantizar el acceso del currículo y el aprendizaje en el marco de respeto a las identidades culturales de cada estudiante. Por ello las escuelas tienen la obligación de realizar diferentes adaptaciones curriculares; las cuales deben estar de acuerdo a los conocimientos, habilidades y destrezas iniciales de los estudiantes que pertenecen a esta institución educativa. Estas adaptaciones deben ser planteadas por los actores educativos de las instituciones educativas respondiendo a las necesidades diagnosticadas en el espacio escolar (Poblete, 2018).

El incremento inmigratorio que se está dando en los distintos países; extiende la necesidad de buscar nuevas oportunidades para los niños y adolescentes en edad escolar; esta búsqueda también va ligado al proceso de elección escolar (Joiko \& Vázque, 2016). Lo cual permite analizar dicho proceso desde dos puntos de vista, desde la inclusión y desde la exclusión, hacia la implementación de un proceso que pueda ser potencialmente inclusivo que permita a todas las personas insertarse en el sistema educativo y a la sociedad, ser aceptado desde sus propias características y experiencias.

El primer encuentro entre las familias y el sistema educativo; el proceso de elección se considera como la correcta toma de decisiones de las familias en un nuevo contexto, en donde está en juego sus identidades. Pero este proceso de elección es vulnerado debido a las exigencias administrativas de cada institución educativa y del propio Mineduc (Joiko \& Vázque, 2016).

La acogida a los jóvenes inmigrantes al sistema educativo se ha verificado a través de la planificación, organización, control, y la inclusión de los niños y jóvenes inmigrantes en los diferentes planteles educativos y garantizando el éxito escolar, permitiendo un enfoque pluricultural. La educación para los inmigrantes es concebida desde diferentes ámbitos: estatal y civil para poder evidenciar si existe una relación con los documentos establecidos y la realidad que se vive (Jiménez \& Valdivia \& Valdés \& Hernández, 2017).

Jiménez \& Valdivia \& Valdés \& Hernández (2017) afirman que la situación de la diversidad cultural en las instituciones es con el fin de cultivar entre la sociedad una justicia cultural, proteger los derechos y que existiera la igualdad de condiciones para todos los estudiantes independiente de su país natal; de esta forma se conoce como la 
sociedad mira a los estudiantes migrantes dentro del sistema educativo y como esto ha ayudado a la incorporación a las aulas. Por otra parte, se ha dejado de lado la preparación de los docentes para abordar y ayudar a la integración de los estudiantes con respecto a sus compañeros y si ellos han realizado adaptaciones en sus planificaciones para contribuir con el fortalecimiento de su cultura.

En este sentido el interés de Estados Unidos y México por la atención y el bienestar de los niños migrantes, han tratado de generar una educación basada en la inclusión, la igualdad de oportunidades y la interculturalidad a través de adaptaciones curriculares, pero solo se ha reflejado una exclusión y por siguiente un fracaso escolar de quienes no cumplen con los parámetros y estándares establecidos en las diferentes instituciones. (Franco, 2017).

Los programas educativos, acuerdos y procesos escolares diseñados para atender las diversas problemáticas de integración por parte de los niños migrantes en Estados Unidos, no son lo suficientemente fuertes como para contrarrestar a la exclusión y segregación por la comunidad educativa y por ende la sociedad; mientras que en México el estudiante se acopla a las normativas del sistema, lo cual indica que los docentes no cubren con las necesidades educativas que presentan los estudiantes.

La migración juvenil hacia los Estados Unidos de América es percibida desde la vulnerabilidad y las diferentes estrategias que las familias utilizan para sobrevivir, para enfocar la relación entre el capital humano, el capital educativo y migración de jóvenes. (Nájera Aguirre \& Herná, 2009)

Las bajas calificaciones de los jóvenes emigrantes, se debe mayoritariamente a jóvenes sin educación básica o que tuvieron una ruptura muy prolongada de sus estudios y se observa una acumulación de discriminación social sobre su lugar de origen. Las familias migrantes influyen en la decisión de estudiar. En los jóvenes principalmente, afirmando que no solo es necesario de recursos educativos, como escuelas, docentes y programas de inclusión, sino también la voluntad familiar, incentivar a los hijos a estudiar, ya que la mayor parte de los adolescentes y jóvenes migran para trabajar.

Otro tema de discusión es la tolerancia que existe en la sociedad y como los grupos mayoritarios trata o visualizan a los migrantes y esto debe ser atendido por las políticas públicas en educación. Aceptar la relación entre modernidad y tolerancia en cuanto se refiera al cumplimiento de los derechos que se encuentran prescritos y también el abordaje de cómo lo anteriormente mencionado influye en la sociedad con el tema de la educación de las personas migrantes. Muñoz Perez, Alarcón Leiva, \& Sanh (2017)

Se manifiesta la iniciativa que surge por cumplir los derechos que tienen todas las personas, aunque sean inmigrantes, como es el acceso, la permanencia, y la no discriminación en las diversas instituciones educativas. Aunque se da poca relevancia a la inclusión desde las aulas y el trato que reciben desde sus docentes los niños y jóvenes estudiantes migrantes. 
Bustos \& Gairín (2017), concuerda con los demas autores que existe una limitada predisposición a la interculturalidad, también que los docentes tienen uno forma de pensar donde los estudiantes se acoplan al sistema escolar y que de ellos depende su rendimiento escolar, por esta razón, no velan por problemas que tienen los estudiantes con el ritmo, conocimientos previos y peor aun adaptaciones curriculares.

La adaptación académica de estudiantes migrantes se manifiesta en los centros educativos, puesto que se verifica la inclusión, dejando a un lado todos los estereotipos. Es necesario conocer la realidad a partir de las experiencias de las personas que han participado en el proceso de adaptación escolar, con el fin de saber cuáles estrategias benefician el éxito académico y dan respuesta a la problemática que surgen en la zona fronteriza.

Las experiencias de exclusión que han vivido los estudiantes migrantes, han generado en ellos una desconfianza y no sentirse plenamente a gusto en sus estudios. Las experiencias de los inmigrantes al tratar de asistir a una escuela es casi una tarea imposible, considerando un factor que se presenta muy a menudo. La mayor razón de inasistencia de los estudiantes a los diferentes centros de educación es debido a la actividad laboral de los padres; debido al problema económico que atraviesan y esa necesidad de aportan en su hogar. (Frades, 2016)

Se debe tener claro que la inmigración no se habla solo en los últimos tiempos, sino que desde el inicio el ser humano ha emigrado de un lugar a otro, por esto es necesario que al lugar donde residen las personas de otras nacionalidades tenga el mismo derecho. La educación es un derecho mundial que debe estar acorde a la integración intercultural.

En gran medida solo miramos la parte afectada de los estudiantes inmigrantes, pero también existe la realidad de los docentes que tiene que saber adaptar al aula a los niños de bajos recursos o problemas familiares y los niños inmigrantes. Esto refleja las bajas calificaciones en relación con los niños autóctonos. (Frades, 2016)

Otro factor de gran importancia es saber la edad de los padres, los motivos de porque decidieron inmigrar y la condonación económica que tiene la familia en el país actual en el que se encuentra. La inasistencia a clases llega a ser perjudicial para los niños y adolescentes inmigrantes si presentan alguno de los factores anteriormente mencionados; presentarán problemas de retraso por el aprendizaje pues los estudiantes no mantienen un hilo de secuencia con las materias y los temas tratados (Victoria María \& Tovar Luis, 2009).

La sociedad manifiesta que la presencia de los niños en las aulas de clase, llegarían a causar un obstáculo en la educación, aunque las estadísticas señalan que no existe ninguna afectación. A más de ser criticados y en el mayor de los casos menospreciados, los estudiantes inmigrantes llegan a tener problemas en el rendimiento académico, puesto los docentes no generaran un clima apropiado, y no existe la colaboración de los padres por contribuir en la escolarización de sus representados. 
Es preciso conocer la conceptualización de diferentes conceptos que permita entender y comprender sobre la inmigración estudiantil y la adaptación escolar; para lo cual citaremos a diversos autores. Entre ellos tenemos:

La Organización Internacional para las Migraciones (OIM), (2016) definen la inmigración como "el proceso por el cual personas no nacionales ingresan a un país con el fin de establecerse en él” (p. 32).

La inmigración es cuando un individuo deja su país de origen y se traslada a otro, en la actualidad esto ha ido creciendo al punto de considerado como un fenómeno en los países que dan la acogida; la mayor parte de los inmigrantes al trasladarse deben dejar su cultura, sus tradiciones y muchas de las veces debe dejar su manera de pensar para adaptarse a las costumbres y aprender a convivir con la sociedad en donde reside. También podemos decir que la inmigración se da por diferentes factores como la economía, la persecución, por estudios o por un cambio de vida.

El Ministerio de Educación del Ecuador (2016), afirma que:

El currículo es la expresión del proyecto educativo que los integrantes de un país o de una nación elaboran con el fin de promover el desarrollo y la socialización de las nuevas generaciones y en general de todos sus miembros; en el currículo se plasman en mayor o menor medida las intenciones educativas del país, se señalan las pautas de acción u orientaciones sobre cómo proceder para hacer realidad estas intenciones y comprobar que efectivamente se han alcanzado (p.4).

El currículo es aquel que norma la enseñanza guiando a los docentes de un país en que se debe enseñar y como enseñar, este es flexible y abierto, por ello el docente debe adecuarlo a las necesidades de cada estudiante, del grupo y del contexto. Los docentes tienen el papel fundamental de llevarlo a cabo, pero tomando en cuenta al estudiante como el personaje principal del proceso de enseñanza aprendizaje; a partir de ello lograr llegar a todos y cada uno de los estudiantes sin distinción alguna; con múltiples experiencias y contenidos.

El Ministerio de Educación del Ecuador (2016) cita a Orjales Villa, I (1999), quién manifiesta que:

Las adaptaciones curriculares se entienden como las sucesivas adecuaciones que, a partir de un currículo abierto, realiza un centro o un profesor para concretar las directrices propuestas por la administración educativa, teniendo presente las características y necesidades de sus alumnos y de su contexto (p.31).

Las adaptaciones curriculares son aquellos medios proporcionados por los docentes o las instituciones educativas permiten a todos los estudiantes acceder al currículo normal a partir de sus propias habilidades y destrezas. Estas adecuaciones se harán dependiendo del contexto y de la realidad a la cual se enfrenta el estudiante. 
La Organización de las Naciones Unidas para la Educación, la Ciencia y la CulturaUNESCO (2005) en el artículo 5 de la Convención sobre la Protección y Promoción de la Diversidad de las Expresiones Culturales puntualiza sobre la "diversidad cultural"; definiéndose como:

La multiplicidad de formas en que se expresan las culturas de los grupos y sociedades. Estas expresiones se transmiten dentro entre los grupos y sociedades. La diversidad cultural se manifiesta no solo en las diversas formas en que se expresa, enriquece y transmite el patrimonio cultural de la humanidad mediante la variedad de expresiones culturales, sino también a través de distintos modos de creación artística, producción, difusión, distribución y disfrute de las expresiones culturales, cualesquiera que sean los medios y tecnologías utilizados (p.5).

La diversidad cultural es aquello que permite identificar y diferenciar de un grupo a otro, o de una sociedad a otra; y que ayuda en el enriquecimiento de cada ser humano, pero si la recibe desde una visión de respeto y tolerancia.

La presente investigación se fundamenta tanto en las normativas nacionales e internacionales.

La Organización de las Naciones Unidas para la Educación, la Ciencia y la Cultura UNESCO (2016) en la Declaración de Incheon plasma la finalidad de la educación; la cuál es brindar a todas las personas iguales oportunidades para acceder a este servicio. Este derecho se dará independiente mente de cuál sea su sexo, origen étnico, origen nacional, social o cultural; para personas migrantes, o pueblos indígenas. "En particular los que se encuentran en situación de vulnerabilidad o de otro tipo, deben tener acceso a una educación de calidad inclusiva y equitativa y oportunidades de aprendizaje a lo largo de la vida" (p.25).

Se reconoce una educación para todos sin distinción alguna, centrada en la calidad que esta debe ofertar, que permita adquirir aprendizajes que contribuyan en el desarrollo de aptitudes y capacidades para enfrentarse al mundo actual sea cual sea el lugar en donde se encuentren. Pero el centrarse en la finalidad del acceso a la educación de todas las personas, no debe cegar otros procesos que deben ir de la mano y se los debe tomar encuentra una vez que ingresan al Sistema Educativo; como son prestar atención sobre si los estudiantes están aprendiendo y si adquieren las competencias pertinentes. Solo si se cubre con todos esos aspectos se podrá alcanzar la educación de calidad para todos.

También nos habla sobre los establecimientos y los programas educativos; los establecimientos deben contar con instalaciones seguras y de fácil acceso; los programas educativos deben ser pertinentes, guiados por enfoques pedagógicos dinámicos y de colaboración centrados en los estudiantes; representados por docentes, establecimientos y los programas educativos versátiles y adaptables; los establecimientos deben contar con instalaciones seguras y de fácil acceso; los programas educativos deben ser pertinentes, guiados por enfoques pedagógicos dinámicos y de colaboración centrados en los 
estudiantes. Los sistemas educativos deben ser pertinentes y adaptables ante todas las necesidades que se puedan presentar y ante los avances tecnológicos; deberán ofrecer medios materiales o humanos que doten de oportunidades amplias y flexibles a los estudiantes para adquirir múltiples aprendizajes a lo largo de su vida, deben definir sus normas o planes de estudio en función del contexto y en consideración de las aptitudes, capacidades y conocimientos iniciales de los estudiantes sus valores o cultura.

La universalidad en la educación es hacia donde se direcciona el trabajo de la Declaración de Incheon. Para ello la UNESCO (2016) plantea que será necesario “movilizar esfuerzos nacionales, regionales y mundiales orientados a: establecer alianzas eficaces e inclusivas, mejorar las políticas educativas y su funcionamiento conjunto; garantizar sistemas educativos altamente equitativos, inclusivos y de calidad para todos; movilizar recursos para financiar correctamente la educación" (p.31).

Entre uno de los principios que reconoce la UNESCO (2016) es que la educación es "un derecho humano fundamental y un derecho habilitador, para hacerlo realidad, los países deben garantizar un acceso universal e igualitario a una educación y un aprendizaje inclusivos y equitativos de calidad, que deberán ser gratuitos y obligatorios"(p.28). Permitiendo así la universalidad de la educación sin dejar a ninguna persona fuera del sistema.

Por ello todos los países deben dar mayor importancia a los procesos educativos mejorando sus políticas y planes. Deben efectuar los cambios que convengan en las estrategias, enfoques, contenidos, estructuras que permitan eliminar barreras sociales, culturales y económicas; liminar aquellas barreras que privan a niños, adolescentes y adultos al derecho a la educación y a un aprendizaje de calidad, remediando así la situación de miles de personas excluidas permitiendo así crear lazos entre continentes, países, regiones, ciudades o comunidades. Los sistemas educativos "deben adaptarse a sus necesidades, independientemente de su origen o procedencia, deben responder a la necesidad de resiliencia de las sociedades y adaptarse a la migración y los desplazamientos" (UNESCO, 2018, p.11).

La Constitución de la República del Ecuador en el artículo 9 manifiesta que las personas extranjeras son consideradas como parte del estado, con el fin de que aquellas personas gocen de los deberes y derechos que se rigen en el Estado.

Otro artículo que respalda a los inmigrantes que se encuentra en el país es el artículo 3, dando a conocer que ninguna persona puede quebrantar los deberes y derechos que ellos tienen al encontrarse dentro del país. Señala en litera 1 y 3 que pueden hacer uso de los diferentes beneficios que brinda el país.

El artículo 11 de la Constitución del Ecuador rige el principio en el literal 2, que dice que no puede existir discriminación a todos los seres humanos que viven dentro del estado ecuatoriano incluyendo a las personas migrantes. 
La Constitución del Ecuador también respalda los siguientes artículos 27 y 28 donde los niños y jóvenes migrantes pueden acceder al sistema educativo del Ecuador, ya que la educación es un derecho universal centrado en el ser humano y no existe discriminación en ninguno de los niveles. Por último, se puede señalar diversos artículos que respaldan el goce de los derechos y la aplicación de los deberes de las personas inmigrantes en igualdad como un ciudadano ecuatoriano, donde el Estado garantizara su seguridad, libertad y trato igualitario a todas las personas que vivan dentro del país, es decir todas las personas se rigen a un mismo sistema. Estos artículos son el art. 66, 40, 45,392 y 416.

En la Ley Orgánica de Educación Intercultural (LOEI) el artículo 5 respalda a las personas migrantes para poder acceder al sistema educativo gratuito como un derecho que debe ser respetado y salvaguardado.

Visto ya desde este punto la educación es un derecho de todas las personas que viven en el Ecuador; siendo así un aspecto universal que todo estado debe garantizar a todos los niños, niñas y adolescentes. El artículo 2 de los principios nos habla de la universalidad y flexibilidad de la educación; universalidad siendo un deber ineludible e inexcusable del estado garantizarlo y flexibilidad que debe adecuarse a las diversidades.

En el Reglamento de la Ley Orgánica de Educación Intercultural (RLOEI); capítulo IV de la educación en situaciones excepcionales podemos ver que el Estado respalda y garantiza la educación de los niños, niñas y adolescentes ya que en el artículo 234 vemos que son personas vulnerables y necesitan tener un trato preferente. En el art. 234 nos habla sobre la situación de vulnerabilidad. Se consideran en situación de vulnerabilidad las personas que se encuentran en condiciones de: literal 8. ser hijos de migrantes con necesidad de protección" (Ministerio de la Educacion, 2017).

También podemos ver que el articulo 235 respalda el trato preferencial a los niños y jóvenes migrantes a tener una educación de calidad de la calidez y en especial inclusiva. El articulo 236 evidencia que no hay impedimento para poder matricular a los niños y jóvenes migrantes, ya que el Sistema Nacional de Educación facilita la obtención de un cupo con otro tipo de documento.

En el reglamento a la LOEI se observa que en el artículo 243 la interculturalidad, habla sobre como favorecer a las diferentes comunidades y cultural a disminuir el racismo y comenzar la inclusión en todas las instituciones del Ecuador.

Cabe recalcar como el Estado ecuatoriano ayuda a los migrantes a continuar sus estudios en el caso de no haber concluido todos los niveles y poder continuar el mismo ciclo de educación es decir que no repita ningún nivel. Por último, se observa en el art 167 el Sistema Educativo Nacional ayuda a la reubicación de los estudiantes extranjeros, con el fin de ayudar a terminar sus estudios, en el caso que no existiera papeles que garanticen el año que deben estar o la culminación de sus estudios.

En el Plan Nacional de Desarrollo toda una vida; el Ecuador busca que prevalezca la 
equidad, la justicia social y la igualdad de oportunidades en todos los niños, niñas y jóvenes sin importar el origen de su nacionalidad, con el fin de que puedan hacer uso de los servicios ecuatorianos públicos para alcanzar la vida que desea en armonía individual, social

La Ley Orgánica de Movilidad Humana, mediante el art 60 nos explica la relevancia de la terminología residencia temporal; la cual es una condición inminente de una persona que migra hacia otro lugar que no es su residencia. Pero también cita que su estadía no debe pasar 2 años esto ya enmarcándonos en el territorio ecuatoriano. Que solo se la podrá renovar una sola vez.

En el marco de lo educativo y concretamente de los estudiantes el permiso recae conforme sea la duración de estudio en cualquier nivel o a su vez practica pre profesional.

El Plan Nacional de Movilidad Humana en la política 2, menciona el fortalecimiento de los derechos de una población que se encuentra en vulnerabilidad y movilidad humana lo que representa un apoyo mutuo entre sociedades y la no discriminación de ninguna índole hacia otra nacionalidad.

En Educación, menciona los cambios que se dan en la sociedad correspondiente a la migración. Planteando la problemática sobre la finalización de sus estudios en sus lugres de origen por tanto prioriza la creación de mecanismos que como educación a distancia, aulas virtuales y plataformas que de alguna manera beneficien a este sector para la finalización de sus estudios. Así también muestra su preocupación hacia las personas que retornan al país para garantizar su reinserción a la educación.

No descarta un inconveniente común que es la validación de títulos profesionales o certificados obtenidos en otros países y viceversa. Pero si asume el compromiso de mejora conforme a gestiones que ayuden a superar este obstáculo.

El Decreto Ejecutivo N.- 1585 afirma que nuestro país es rico en pueblos y nacionalidades y el estado ecuatoriano es consciente de esto por tanto mediante políticas públicas se compromete al apoyo incondicional hacia este sector y decreta apoyar y fortalecer el sistema de educación intercultural bilingüe a través de la autoridad educativa nacional que formulara dichas políticas para el sistema educativo.

\section{Metodología.}

El método empleado para la recolección de la información es el método cuantitativo mediante la encuesta como técnica y el cuestionario como el instrumento. Se empleó el instrumento desarrollado y aplicado por Valero José Ramón, (2002 ); las preguntas que se tomó son 13 preguntas para docentes y 7 para estudiantes; las cuales fueron seleccionadas y adaptadas dependiendo de nuestra necesidad y de nuestro contexto mediante el método de análisis-síntesis. La encuesta consta de preguntas cerradas de elección única (politómicas); las cuáles se aplicó en la ciudad de Ambato en la Unidad Educativa "Teresa Flor", jornada matutina, los sujetos investigados fueron 6 docentes y 
18 estudiantes inmigrantes con el fin de indagar la inmigración estudiantil y la adaptación en el sistema educativo.

\section{Resultados.}

Al realizar la tabulación de las encuestas aplicadas a los docentes de la Unidad Educativa "Teresa Flor ", se obtuvo los siguientes resultados:

Respondiendo a la pregunta ¿Los padres de los alumnos inmigrantes, en general, se encuentran en el país en situación de? los docentes manifiestan que el $33 \%$ de los padres se encuentran regulares en el país y el $67 \%$ de los padres se encuentran irregulares. Se deduce que la mayor parte de los padres son irregulares en el país; lo que indica que puede existir repercusión en el rendimiento académico del estudiante y en la adaptación escolar puesto que no existe una estabilidad en el ámbito familiar y en cualquier momento podrían ser deportados.

Gráfico 1. Situación regular o irregular en el país.

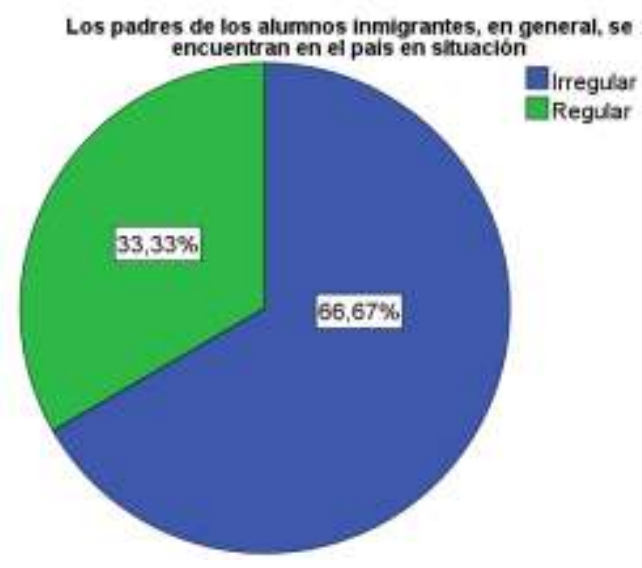

Fuente: Elaboración autores.

Ante la interrogante ¿los estudiantes inmigrantes incorporados al sistema escolar, se integran en el centro como cualquier otro estudiante?; manifiestan que el 17\% siempre se integran en el centro y el $83 \%$ manifiestan que a veces. Reflejando así que muestran dificultad en incorporarse puesto que existe un choque de culturas que repercute en la convivencia. En cuanto a la pregunta relacionada con ilos estudiantes inmigrantes incorporados al sistema escolar, tienen problemas de adaptación? el 100\% manifiesta que a veces; deduciendo que existen estudiantes que se adaptan rápidamente al entorno, entablan relaciones intrapersonales con facilidad y comprenden a sus docentes; mientras que se puede evidenciar que existen estudiantes que se les dificulta adaptarse debido a la falta de valores lo que causa indisciplina en el aula y a la falta de conocimientos en cuanto a los contenidos que ya deberá dominar en relación a su edad. 
Gráfico 2. Integración de los estudiantes inmigrantes en el centro.

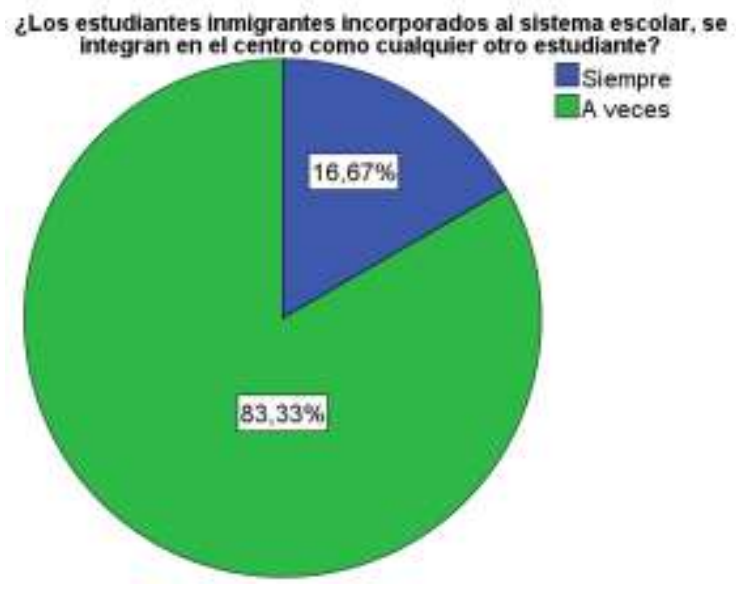

Fuente: Elaboración autores.

Gráfico 3. Adaptación de los estudiantes inmigrantes en el centro.

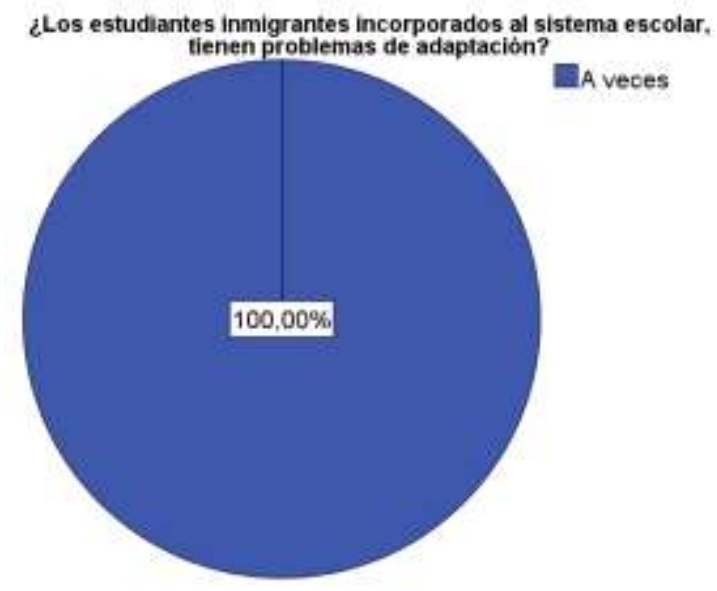

Fuente: Elaboración autores.

En cuanto a la pregunta ¿ser inmigrante influye de manera negativa en el rendimiento académico? el $66 \%$ manifiesta que bastante, el $17 \%$ poco y el $17 \%$ nada. Lo que indica y ser inmigrante influye en su mayoría en el rendimiento académico ya que los docentes afirman que los estudiantes inmigrantes especialmente los estudiantes venezolanos presentan un grado de dejadez en cuanto a la realización de las tareas, también manifiestan que no les gusta trabajar en clases y faltan muy seguido a clases y esto es algo que dificulta en su aprendizaje, ya que llegan a faltar por semanas y esto no contribuye en su rendimiento académico, generan vacíos en cuanto a los conocimientos de la materia lo cual no permite al docente; repercutiendo así en el rendimiento académico. En relación a la interrogante ¿el rendimiento escolar de estudiantes inmigrantes, está condicionado por la edad de acceso al sistema educativo ecuatoriano? el 67\% manifiesta que bastante y el $33 \%$ que poco; se deduce que la edad de acceso si influye en el rendimiento académico puesto que de esto dependerá que el niño se adapte rápidamente al entorno y a las normas y reglas del centro escolar. 
Gráfico 4. Influencia de ser inmigrante en el rendimiento académico.

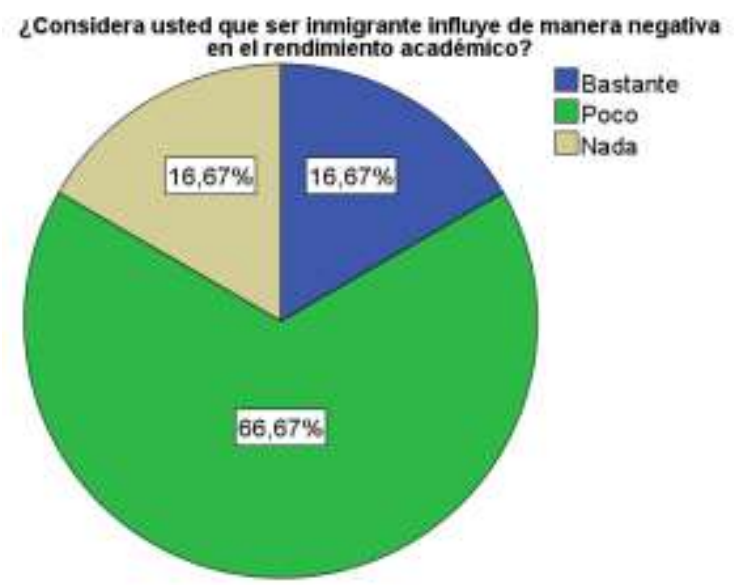

Fuente: Elaboración autores.

Gráfico 5. Relación entre la edad de acceso y el rendimiento escolar.

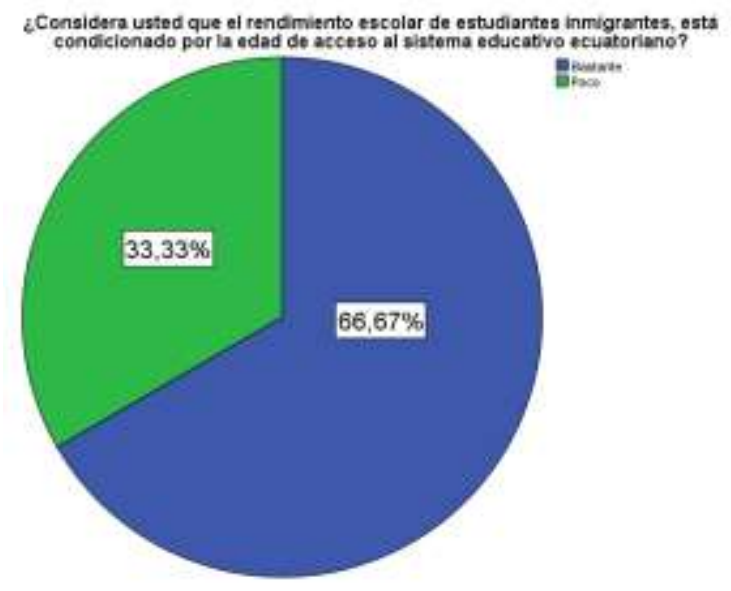

Fuente: Elaboración autores

Ante la interrogante ¿los estudiantes inmigrantes protagonizan peleas dentro de la institución? el $17 \%$ afirma que siempre, el 50\% a veces y el 33\% nunca; lo que indica que los estudiantes inmigrantes son más propensos a protagonizar peleas con sus compañeros o a formar indisciplina dentro del aula; los docentes afirmar que en muchos de los casos estas peleas se dan porque existe una creencia de superioridad de los estudiantes inmigrantes ante los estudiantes ecuatorianos. En cuanto respondiendo a la pregunta ¿los estudiantes inmigrantes se adaptan a las normas de convivencia y las formas de vida del contexto? el $17 \%$ manifiesta que siempre y el $83 \%$ a veces; lo que indic a que lo estudiantes inmigrantes tienen dificultad en adaptarse a las normas de convivencia del entorno puesto que difiere en su mayoría a las normas del país de procedencia. En relación a la interrogante ¿los padres y/o madres de los alumnos inmigrantes? el 17\% manifiestan que los padres visitan al tutor por iniciativa propia, el $67 \%$ sólo acuden al ser llamados por el propio tutor y el 17\% se desentienden del centro; lo que indica que los padres no están al pendiente de sus hijos y no muestran interés en cuanto al rendimiento y 
adaptación de su hijo, no busca la forma para trabajar en conjunto con el docente.

Gráfico 6. Estudiantes inmigrantes y peleas.

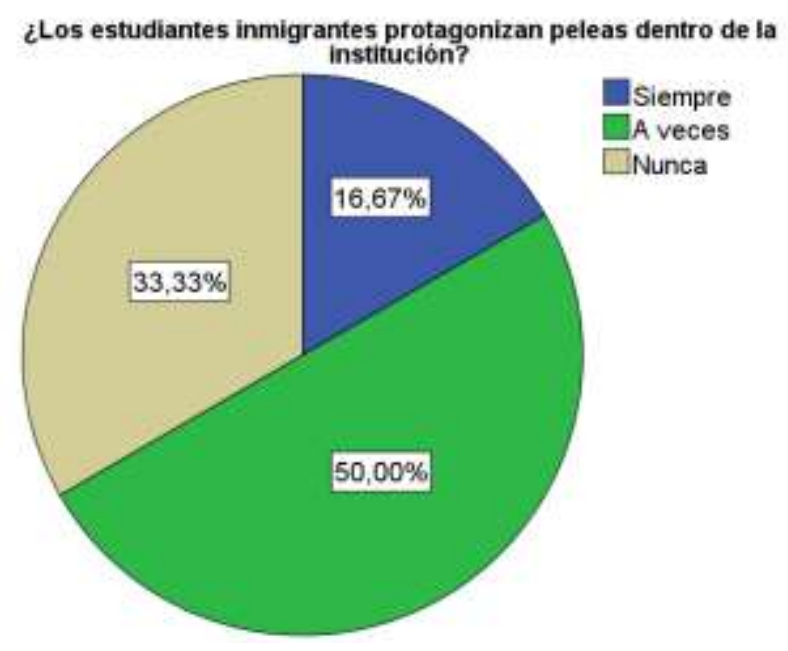

Fuente: Elaboración autores

De la encuesta aplicada a los estudiantes se obtuvo los siguientes resultados:

De los estudiantes inmigrantes que existen en la institución el $67 \%$ proceden de Venezuela, el 22\% de Colombia y el 11\% de España. En relación a la pregunta de ¿En qué año empezó a vivir en el Ecuador?; el 11\% manifiesta que entre el año 2015 - 2016, el 33\% en el 2017, el 56\% entre el año del 2018 e inicios del 2019. Se deduce que la mayoría de los estudiantes inmigrantes son venezolanos y que inmigraron de su país durante la crisis financiera que azotó a su país y obligó a muchas familias a buscar nuevos rumbos para poder salir adelante.

Gráfico 7. País de procedencia.

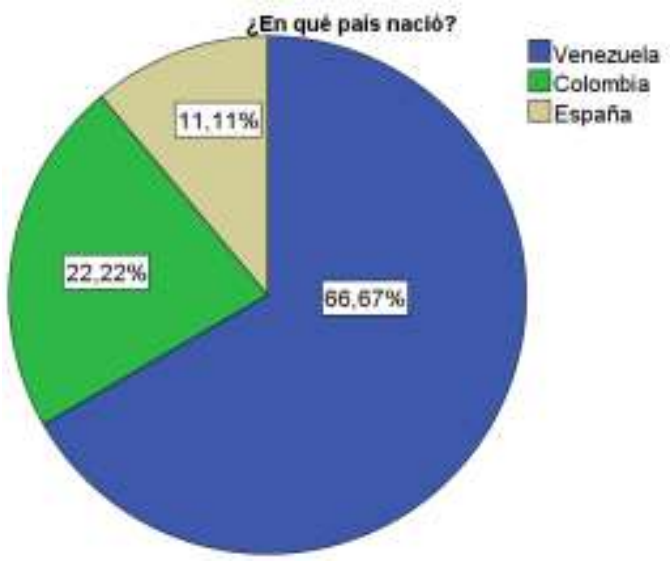

Fuente: Elaboración autores 
Gráfico 8. Año de ingreso al país.

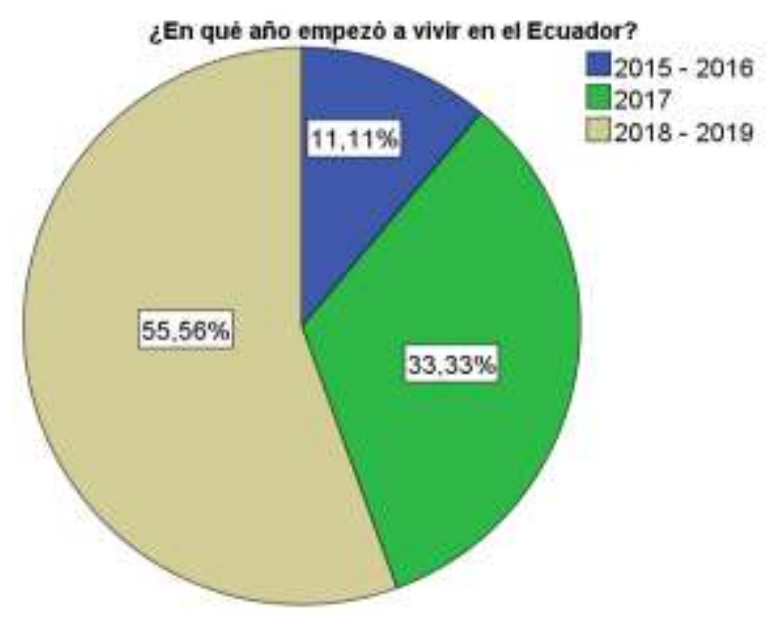

Fuente: Elaboración autores

\section{Conclusiones.}

- Según los resultados encontrados se concluye que a los niños, niñas y adolescentes en edad escolar se les dificulta integrarse al centro escolar; puesto que existe un choque de culturas y por ende problemas con los docentes y compañeros; aspectos que repercute en el rendimiento académico. También a esto se suma la falta de responsabilidad de los padres para trabajar en conjunto con los docentes para lograr que su hijo se sienta a gusto; puesto que no acuden al centro por voluntad propia en busca de soluciones o para conocer los medios que los docentes plantean para lograr la integración de sus representados.

- En el ámbito de las relaciones interpersonales es una fuente de conflicto especialmente por los estudiantes venezolanos; pues se alude que dichos estudiantes son los que en menor grado se relacionan con sus compañeros. En este caso se observa que se están construyendo percepciones en relación con la nacionalidad presenciando explícitamente prejuicios y estereotipos que subvaloran el desempeño escolar de los estudiantes venezolanos

- Finalmente, es necesario recalcar que la educación es un derecho fundamental, básico e indispensable que debe ser garantizado y salvaguardado por el estado; para que todas las personas sin discriminación alguna tengan acceso. Este derecho también es aplicable para las personas inmigrantes por ende los centros educativos deben contar con docentes capacitados e infraestructura adecuada que logre su desarrollo social, cognitivo y afectivo; mediante el desarrollo de diferentes adaptaciones que permita ser respetado y desarrollarse desde sus propias convicciones; pero al mismo tiempo respetando, comprendiendo y aprendiendo las diversas costumbres o convicciones que se presentan en el contexto en el que se encuentran. 


\section{Referencias Bibliográficas}

Asamblea Constituyentes. (2017). Constitución del Ecuador. Quito. Obtenido de Constitución de la República del Ecuador.

Ávalos, B. (2012). Inmigrantes en Chile: límites y potencialidades de los niveles locales para construir un nuevo pacto de integración social.

Bustos, R., \& Gairin, J. (2017). La adaptación académica de estudiantes migrantes en contexto de frontera. Calidad en la educación. Obtenido de https://scielo.conicyt.c1/scielo.php?script=sci_abstract\&pid=S071845652017000100193\&lng=es\&nrm=iso

Carabaña, J. (2008). El impacto de la inmigración en el sistema educativo español. Real Instituto Elcano, 11.

Cerón, L., Pérez Alvarado, M., \& Poblete, R. (2017). Percepciones Docentes en torno a la Presencia de Niños y Niñas Migrantes en Escuelas de Santiago: Retos y Desafíos para la Inclusión. Revista latinoamericana de Chile. Obtenido de https://scielo.conicyt.cl/scielo.php?script=sci_abstract\&pid=S0718$73782017000200015 \& \operatorname{lng}=$ es\&nrm=iso

Consejo Nacional De Planificación. (2017). Plan Nacional de Desarrollo.

Dominguez, C. (20 de enero de 2014). SCRIBD. Obtenido de https://es.scribd.com/document/200482438/Integracion-educativa

Frades, S. (2016). Inmigración y educación: un relato abierto más allá de lo escolar. Avances en Supervición Educativa.

Franco, M. (2017). Sistemas educativos y migración. Una mirada a la educación en Estados Unidos y México. Scielo.

Hernández Yulcerán, A. (2016). El currículo en contextos de estudiantes migrantes: Las complejidades del desarrollo curricular desde la perspectiva de los docentes de aula. Estudios pedagógicos (Valdivia). Obtenido de https://scielo.conicyt.cl/scielo.php?script=sci_arttext\&pid=S071807052016000200009

Jiménez \& Valdivia \& Valdés \& Hernández. (2017). Migración y escuela: Análisis documental en torno a la incorporación de inmigrantes al sistema. Psicoperspectivas: Individuo y sociedad, 16. Obtenido de http://www.psicoperspectivas.cl/index.php/psicoperspectivas/article/viewFile/94 $0 / 608$ 
Joiko, S., \& Vázque, A. (2016). Acceso y elección escolar de familias migrantes en Chile: "No tuve problemas porque la escuela es abierta, porque acepta muchas nacionalidades". Calidad en la educación.

Ministerio de Educacion. (2015). Ley Orgánica de Educación Intercultural. Quito.

Ministerio de Educación. (2016). Currículo de los niveles de educación obligatoria.

Obtenido de

https://educacion.gob.ec/wpcontent/uploads/downloads/2016/08/Curriculov2.pdf

Ministerio de la Educacion. (2017). RLOEI. Quito.

Miniterio de Educación. (2016). Guía para la implementación del currículo del área de Ciencias Naturales en el subnivel elementa. Obtenido de https://educacion.gob.ec/wp-content/uploads/downloads/2017/02/Guia-deimplementacion-del-Curriculo-de-CCNN-1.pdf

Muñoz Perez, E., Alarcón Leiva, J., \& Sanh, S. (2017). Modernidad, tolerancia y migración: consecuencias para la educación en Chile. Scielo, 39, 256-778. doi:10.1590/ES0101-73302018178659

Nájera Aguirre, J. N., \& Herná, J. M. (2009). Educación y migración juvenil hacia estados unidos de américa.

Obtenido

de http://www.comie.org.mx/congreso/memoriaelectronica/v10/pdf/area_tematica_ 10/ponencias/1161-F.pdf

Organización Internacional para las Migraciones. (2016). Glosario sobre migraciones. (D. G. Voluntariado., Ed.) Obtenido de https://publications.iom.int/system/files/pdf/iml_7_sp.pdf

Poblete, R. (2018). El trabajo con la diversidad desde el currículo en escuelas con presencia de niños y niñas migrantes: Obtenido de Revista Cielo: http://www.scielo.org.mx/pdf/peredu/v40n159/0185-2698-peredu-40-159-51.pdf UNESCO. (20 de octubre de 2005). Convención de la UNESCO sobre la Protección y Promoción de la Diversidad de las Expresiones Culturales. Obtenido de https://unesdoc.unesco.org/ark:/48223/pf0000142919_spa

UNESCO. (2016). Educación 2030 Declaración de Incheon. Obtenido de http://unesdoc.unesco.org/images/0024/002456/245656s.pdf

UNESCO. (2018). Informe de Seguimiento de la Educación en el Mundo 2019. Obtenido de Migración, desplazamiento y educación: CONSTRUYENDO PUENTES, MUROS: http://unesdoc.unesco.org/images/0026/002659/265996s.pdf 
Valero Escanden , J. R. (2002 ). INMIGRACIÓN Y ESCUELA: La escolarización en España de los hijos de los. Publicaciones de la Universidad de Alicante.

Victoria María \& Tovar Luis. (2009). Migración internacional y educación: una aproximación al efecto sobre la asistencia escolar en Colombia. Cuadernos de Economía, 175-204.

Zúñiga, V. (2013). Migrantes internacionales en las escuelas mexicanas: desafíos actuales y futuros de política educativa. Sinética. Obtenido de https://www.redalyc.org/articulo.oa?id=99827467009

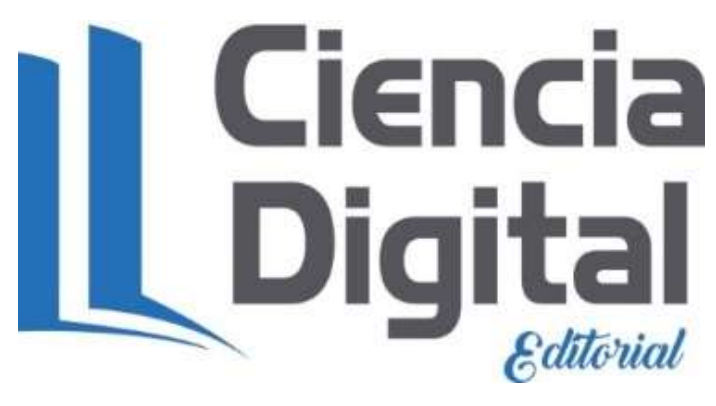




\section{Para citar el artículo indexado.}

Pimbo A., Canchignia A., Paredes N. \& Yungán R. (2019). La inmigración estudiantil y la adaptación escolar. Revista electrónica Ciencia Digital 3(2), 198-217. Recuperado desde:

http://cienciadigital.org/revistacienciadigital2/index.php/CienciaDigital/article/view/385 $\underline{1833}$

\section{Liencia}

El artículo que se publica es de exclusiva responsabilidad de los autores y no necesariamente reflejan el pensamiento de la Revista Ciencia Digital.

El artículo queda en propiedad de la revista y, por tanto, su publicación parcial y/o total en otro medio tiene que ser autorizado por el director de la Revista Ciencia Digital.
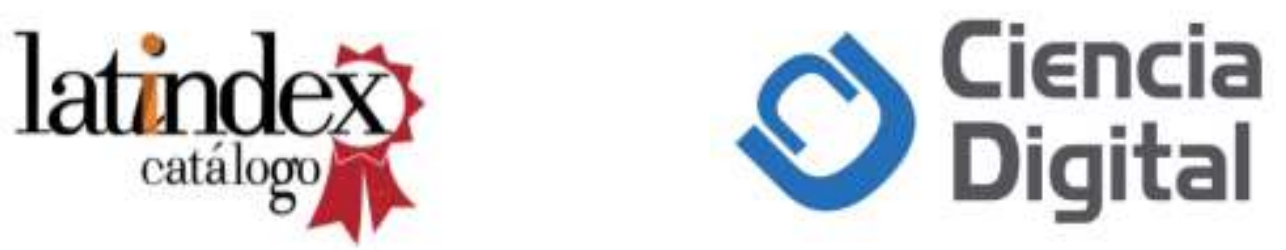